\title{
Magnetic Equivalent Circuit Modeling of an Axial-Field Magnetic Gear
}

\author{
G. E. Ruiz-Ponce, M. A. Arjona and C. Hernández \\ Graduate and Research Center, Tecnológico Nacional de México, Campus Laguna, Torreón, Coah 27000, México
}

\begin{abstract}
This paper presents a two-dimensional (2D) magnetic equivalent circuit (MEC) model to investigate the magnetic field distribution in the air-gap of an axial-field magnetic gear (AFMG). The MEC model is configured as a meshed reluctance network (RN) with permanent magnet magnetomotive-force sources. The MEC model based on RN is considered as a good compromise between accuracy and computational effort. This is a new model that will allow a faster analysis and design for the AFMG. Flux density in the air-gap is calculated with the proposed model and verified by finite element simulations.
\end{abstract}

Key words: Finite element method (FEM), MEC, magnetic gears, RN.

\section{Introduction}

Magnetic gears (MGs) are increasingly studied as potentially useful tools for an efficient mechanical power transmission without the issues associated to conventional mechanical gears. MGs hold important advantages over their mechanical counterparts. They can realize speed change and torque transmission between input and output shafts by a contactless mechanism with quiet operation and overload protection. The most studied MGs technology is focused on radial-field topology. However, for the application of contactless coupling, the axial-field magnetic gears (AFMGs) are thought to be more practical due to its simpler mechanical structure and can be designed compactly [1]. Mezani et al, [2], investigated one of the early AFMG topologies. Lubin et al [3] developed a two-dimensional (2D) analytical model for the original design of the AFMG. The analysis of the magnetic field distribution in the air-gap is of main importance for predicting and optimizing the performance of MGs; hence, the modeling method is very important in their design

Corresponding author: G. E. Ruiz-Ponce, M.Sc. in electrical engineering, research fields: electromagnetic devices, electrical machines modeling and simulation, and transients in power networks. process. The magnetostatic finite element method (FEM) and the analytical methods are mainly used to carry out the massive calculation to evaluate the magnetic field distribution in the air-gap. However, although these methods have high-level accuracy and can yield excellent results, their attractive use is reduced due to its high computational cost [4]. The magnetic equivalent circuit (MEC) based on reluctance networks (RN) offers an alternative modeling method. This method is a mesh-based circuit representation. With this configuration, more details can be taken into consideration and satisfactory accuracy can be achieved compared to FEM but with only a fraction of computational time. The MEC method can support steady-state and dynamic simulations; it has been proposed as the basis for design optimization [5]. Steady-state characteristics can reveal the main transmitting capability of the MGs. There are only a few published works on MEC modeling applied to MGs [6, 7]. However, these works consider the radial-field topology. This paper presents a steady-state modeling of an AFMG employing a 2D MEC based on RN, using a mesh-flux formulation.

\section{The AFMG Topology}

Fig. 1a shows the selected AFMG topology. This 
topology consists of three main structures: two rotors with axially oriented permanent magnets (PMs) on their surfaces and a modulator with stationary ferromagnetic pole-pieces between the two rotors. The low-speed rotor includes a back iron disk and $p_{l}$ PMs pole pairs; the high-speed rotor includes a back iron and $p_{h}$ PMs pole pairs. The number of the pole-pieces of the modulator is $n_{s}$, which is given by the sum of the pole pairs of the rotors. The two rotors on both sides of the air-gap interact by the magnetic flux axially across the ferromagnetic pole-pieces. We have considered two configurations. The first configuration considers $p_{l}=7$ and $p_{h}=2$, hence, $n_{s}=9$ as in Ref. [3]. The second configuration considers $p_{l}=23$ and $p_{h}=4$, hence, $n_{s}=27$, as in Ref. [2].

\section{2D MEC}

Fig. 1b shows the 2D MEC for the configuration $p_{l}$ $=7$ and $p_{h}=2$. A similar $2 \mathrm{D}$ MEC was constructed for the configuration $p_{l}=23$ and $p_{h}=4$. For both configurations, the AFMG is divided into seven layers in the axial direction to take into account the different materials. The minimum repetitive unit in that direction, i.e. the number of steel pole pieces, determines the number of divisions in the circumferential direction. This is an integer number. Hence, the accuracy of the results is proportional to the number of pole divisions. The model is based on flux mesh equations rather than nodal equations. Under non-linear operating conditions, the computational performance of the mesh-based MEC formulation is superior to that of the nodal-based formulation [8].

The mesh-based $\mathrm{RN}$ is the basis for the MEC formulation. The base geometry form for each reluctance element is a sector layer, where for a 2D modeling, only the axial and circumferential components are considered. Linear reluctance elements are defined only by their geometries. They represent the PMs, the air-gaps and the slots between the pole pieces. Non-linear reluctance elements depend on both the geometry and the magnetic non-linearity of the curve $\mathrm{B}-\mathrm{H}$, corresponding to the ferromagnetic material. They represent the rotors yokes and the ferromagnetic pole-pieces. The sources are represented by the PMs, modeled by magnetomotive force sources functions, (MMF). The overall system is defined in the following form:

$$
[\mathcal{R}(\varphi)] \bar{\varphi}=\bar{F}(\theta)
$$

The solution of Eq. (1) is obtained using Newton-Raphson method:

$$
\begin{aligned}
& \bar{\phi}_{m_{i+1}}=\bar{\phi}_{m_{i}} \\
& +\left[[\mathcal{R}]+\frac{\partial\left[\mathcal{R}\left(\phi_{m_{i}}, \varphi\right)\right]}{\partial \phi_{m_{i}}}\right]^{-1}\left\{F(\theta)-[\mathcal{R}] \bar{\phi}_{m_{i}}\right\}
\end{aligned}
$$

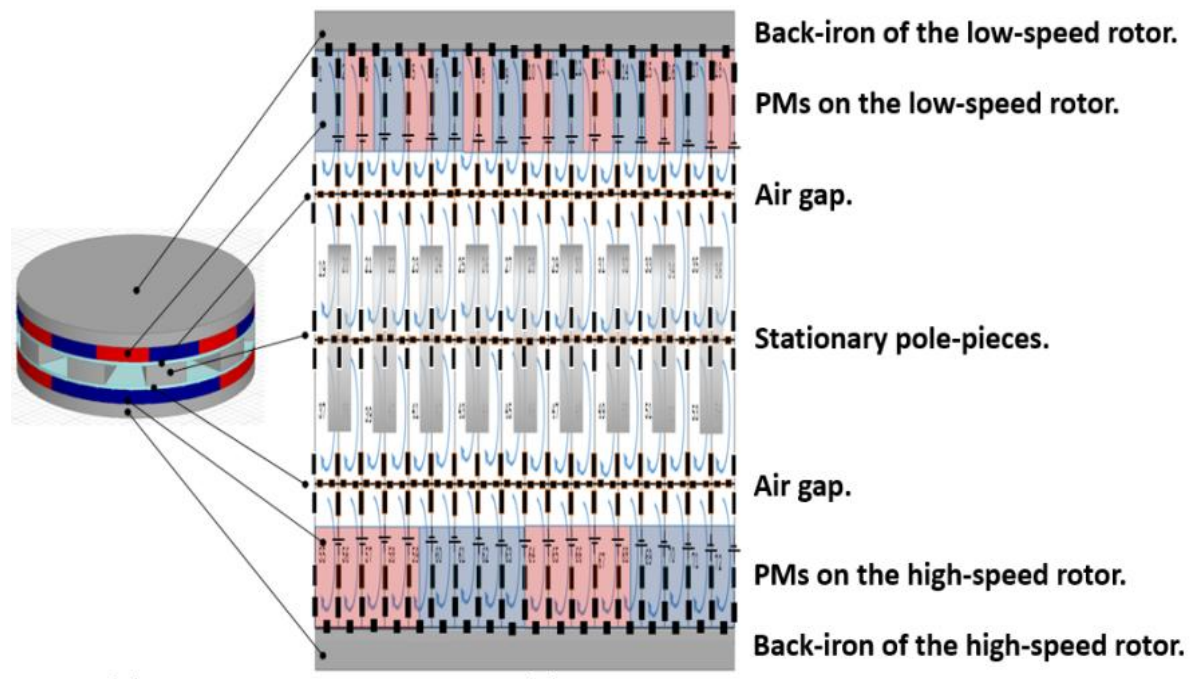

(a)

(b)

Fig. 1 Topology of the AFMG: (a) Structure; (b) 2D MEC. 


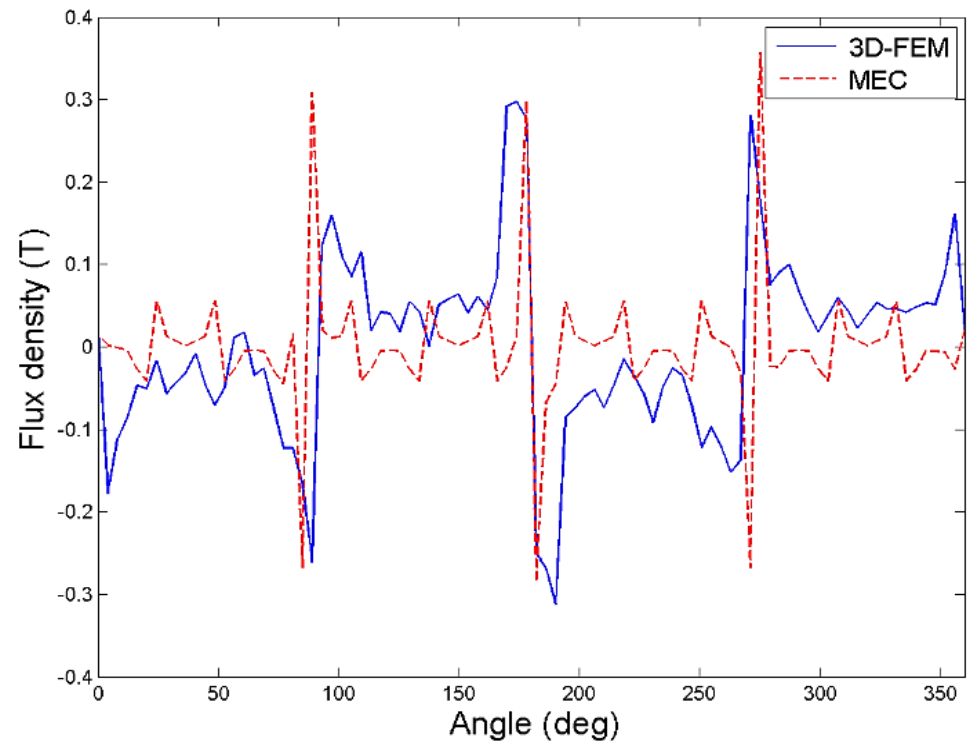

Fig. 2 Axial flux density in the high-speed rotor air-gap: $p_{l}=7$ and $p_{h}=2$.

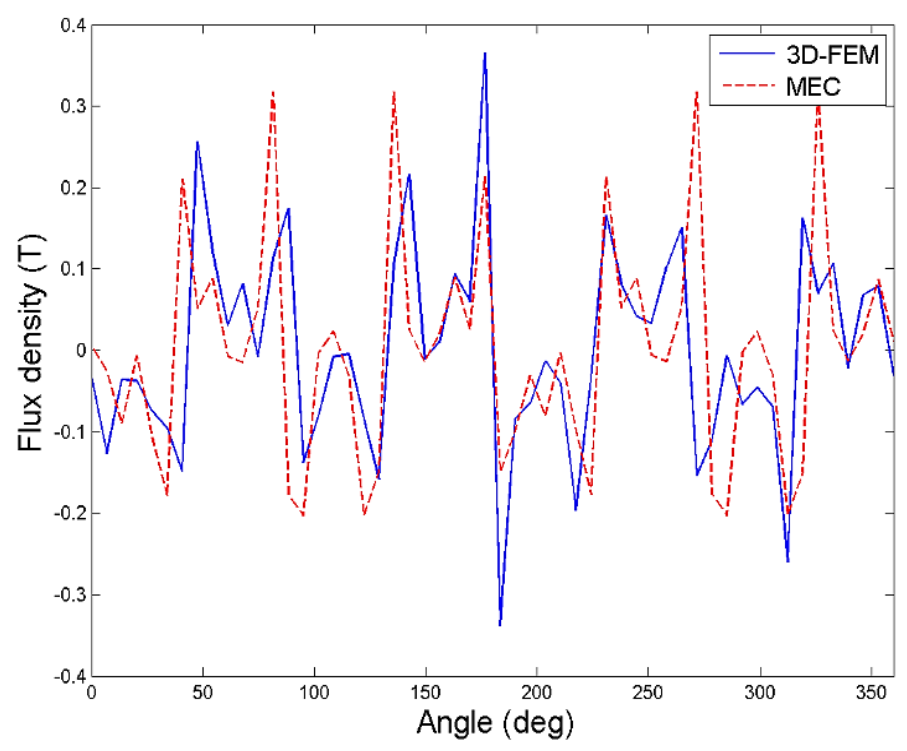

Fig. 3 Axial flux density in the high-speed rotor air-gap: $p_{l}=23$ and $p_{h}=4$.

where $\phi$ is the loop flux vector, $\varphi$ is the branch flux vector, $\mathcal{R}$ is the reluctance element matrix and $F(\theta)$ is the MMF sources which are functions of the rotor position, $\theta$.

\section{Preliminary Results and Validation}

In order to compare the results of the MEC modeling, a 3D finite element analysis (FEA) model was created in the ANSYS Maxwell finite element simulation software package. Figs. 2 and 3, respectively, illustrate the calculated variation of the axial component of the flux density in the middle of the high-rotor air-gap for $p_{l}=7$ and $p_{h}=2$ configuration and $p_{l}=23$ and $p_{h}=4$ configuration. In both plots, it can be found that the modulation of the ferromagnetic pole-pieces on the magnetic field distribution in the air-gap determined in the MEC exhibits a close tendency to that presented by the 3D-FEA model. Currently, we are working with the optimization stage of the MEC model. 


\section{Conclusion}

The MEC modeling allows us to easily parameterize the $\mathrm{RN}$ to represent different topologies of MGs. In this paper, a new model for the axial-type magnetic gear is presented using an MEC based on RN. Magnetic flux density has been calculated and compared to a 3D-FEA model. The results in both models show a close agreement. This demonstrates that an MEC model can predict the complicated distribution of the magnetic field density in the air gap when the parameterization of this model is compatible with FEM model.

\section{References}

[1] Zhu, D., Yang, F., Du, Y., Xiao, F., and Ling, Z. 2016. “An Axial-Field Flux-Modulated Magnetic Gear." IEEE Transactions on Applied Superconductivity 26 (4): 1-5.

[2] Mezani, S., Atallah, K., and Howe, D. 2006. "A High-Performance Axial-Field Magnetic Gear." J. Appl. Phys. 99: 08R303-1.

[3] Lubin, T., Mezani, S., and Rezzoug, A. 2013. "Development of a 2-D Analytical Model for the
Electromagnetic Computation of Axial-Field Magnetic Gears." IEEE Transactions on Magnetics 49 (11): 5507-21.

[4] Zhou, T., Huang, Y., Dong, J., Guo, B., and Zhang, L. 2014. "Design and Modeling of Axial Flux Permanent Magnet Machine with Yokeless and Segment Armature Using Magnetic Equivalent Circuit." In Proc. Of the 17th International Conference on Electrical Machines and Systems (ICEMS), Oct. 2014.

[5] Amrhein, M., and Krein, P. T. 2009. "3-D Magnetic Equivalent Circuit Framework for Modeling Electromechanical Devices." IEEE Transactions on Energy Conversion 44 (2): 397-405.

[6] Fukuoka, M., Nakamura, K., and Ichinokura, O. 2011. "Dynamic Analysis of Planetary-Type Magnetic Gear Based on Reluctance Network Analysis." IEEE Transactions on Magnetics 47 (10): 2414-7.

[7] Wu, Y.-C., and Jian, B.-S. 2014. "Magnetic Field Analysis of a Coaxial Magnetic Gear Mechanism by Two-Dimensional Equivalent Magnetic Circuit Network Method and Finite-Element Method.” Appl. Math. Modell. 39 (19): 5746-58.

[8] Derbas, H. W., Williams, J. M., Koenig, A. C., and Pekarek, S. D. 2009. "A Comparison of Nodal- and Mesh-Based Magnetic Equivalent Circuit Models.” IEEE Trans. Energy Convers. 24 (2): 388-96. 